\title{
Discrimination between strong field molecular ionization pathways using ultrafast pulse shaping
}

\author{
William D. M. Lunden, Dominik Geißler, Péter Sándor, and Thomas C. Weinacht \\ Department of Physics and Astronomy, Stony Brook University, Stony Brook, New York 11794 USA \\ Tamás Rozgonyi \\ Institute of Materials and Environmental Chemistry, \\ Research Centre for Natural Sciences, Hungarian Academy of Sciences, \\ Magyar tudósok körútja 2, Budapest, HU-1117, Hungary
}

\begin{abstract}
We use ultrafast laser pulse shaping to discriminate between different pathways to multiple continua in strong field molecular ionization. Shaping the laser pulse which ionizes the molecule allows us to control the photoelectron spectrum, which we interpret using a newly developed model of resonantly enhanced multiphoton ionization. Our measurements and calculations allow us to distinguish between a single intermediate resonance leading to multiple continua and multiple intermediate resonances each leading to a separate continuum.
\end{abstract}

\section{INTRODUCTION}

Several experiments over the past few years have provided evidence for strong field ionization to multiple continua in small molecules with many equivalent electrons (electrons in orbitals with similar binding energies) [1-5] . Removing an electron from multiple molecular orbitals, which generally corresponds to populating a superposition of cationic molecular states, launches an electron hole wave packet, which is of great interest for understanding attosecond electron dynamics, non-Born Oppenheimer dynamics, electron correlation and the process of strong field ionization itself $[1,3,6-8]$.

Strong field molecular ionization is typically characterized by the Keldysh parameter, $\gamma=\sqrt{\frac{I_{P}}{2 U_{P}}}$, which distinguishes between multiphoton ionization $\gamma \gg 1$, and tunnel ionization, $\gamma \ll 1[9,10]$. Here $\mathrm{I}_{P}$ is the ionization potential and $\mathrm{U}_{P}$ is the ponderomotive energy, or the average energy of an electron oscillating in the laser field. Experiments that are carried out in the tunnel ionization regime can be thought of as operating in the low frequency limit, where the molecular response to the drive field is almost instantaneous, and resonances do not play an important role. In the multiphoton limit, resonances can play an important role in the ionization, enhancing the yield and determining the final state. In particular, in strong field multiphoton ionization, dynamically driven Freeman resonances, which correspond to intermediate neutral states Stark shifting into resonance [11], can dominate the ionization dynamics.

In this paper we examine the role of Freeman resonances in the strong field ionization of a small polyatomic molecule with multiple low lying ionic states. Such a system has comparable binding energies associated with several occupied orbitals, resulting in ionization to multiple ionic states even for the lowest intensities at which ionization occurs. The general question we are interested in addressing is whether resonantly enhanced ionization to multiple ionic states proceeds predominantly through separate resonances for each ionic state or a single intermediate resonance. This is closely related to the question of correlation between initial and final states in strong field ionization, and more generally, the issue of electron correlation in strong field ionization [12]. Here we study strong field ionization of $\mathrm{CH}_{2} \mathrm{BrI}$ and determine if a single resonance is responsible for enhancing the ionization to multiple ionic states, or whether multiple intermediate resonances are involved. Our approach is to study the photoelectron spectrum as a function of laser pulse shape.

Measurements of the photoelectron spectrum for $\mathrm{CH}_{2} \mathrm{BrI}$ and similar molecules (e.g. $\mathrm{CH}_{2} \mathrm{I}_{2}$ and $\mathrm{CH}_{2} \mathrm{BrCl}$ ) show multiple peaks in the photoelectron spectrum, even at the lowest intensities where ionization occurs. These are illustrated in figure 1. Each spectrum in 1 is generated by measuring a two dimensional velocity map image, inverse Abel transforming the data and then plotting the number of electrons measured as a function of their kinetic energy. The peaks can be interpreted in terms of ionization to multiple ionic states of the molecule, leading to photoelectron energies of $\epsilon_{i}=n \hbar \omega-I_{p}^{i}-U_{p}-E_{D S S}^{i}$ [1], where $\mathrm{n}$ is an integer, $\omega$ is the laser radial frequency, and $I_{p}^{i}$ and $E_{D S S}^{i}$ are the ionization potential and dynamic Stark shift of the $i^{t h}$ ionic state. In the limit of negligible Stark shifts for the ionic states, one can roughly determine the ponderomotive shift at which each peak is produced by measuring the photoelectron spectrum as a function of laser intensity. Calculations of the Stark shifts for the ionic states of $\mathrm{CH}_{2} \mathrm{BrI}$ at the peak pulse intensities we used for the measurements described here yield shifts of less than $0.1 \mathrm{eV}$ [13]. Ionization of molecules to the same ionic state via different Freeman resonances can result in separate peaks in the spectrum. However, such peaks cannot be separated by an energy larger than the difference of the ponderomotive shifts associated with their appearance intensities. Furthermore, coincidence measurements, in conjunction with ab initio calculations of the energies of the first five ionic states (detailed in a separate publication [14]), allow us to assign the two 


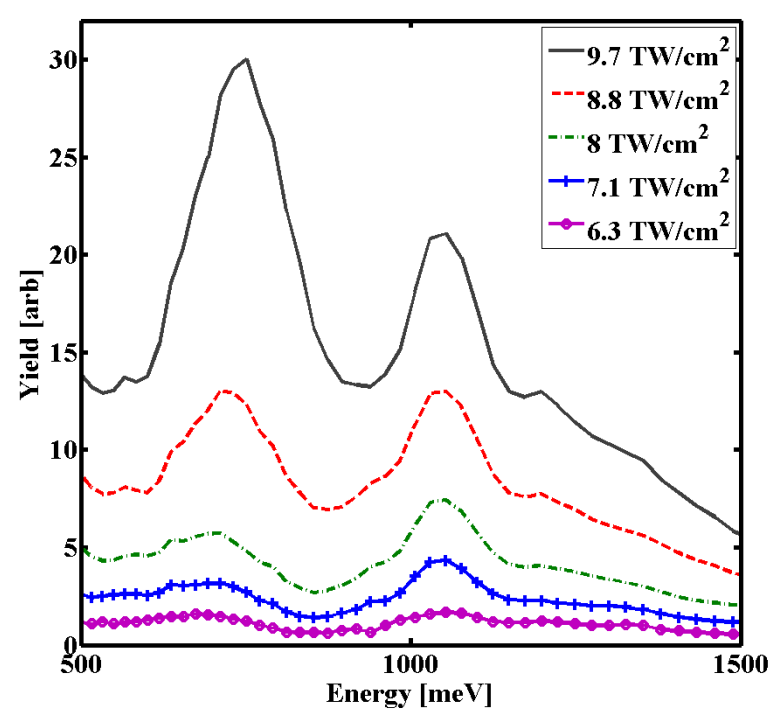

FIG. 1: Photoelectron spectra for ionization of $\mathrm{CH}_{2} \mathrm{BrI}$ with unshaped pulses of different intensity. The intensities are given in the legend.

rightmost peaks in figure 1 (at roughly 1.05 and 1.25 $\mathrm{eV})$ to separate ionic states (electronic continua) of the molecule. As the peaks do not shift in position with intensity, we conclude that they correspond to Freeman resonances, which do not shift ponderomotively since the ionization is predominantly occurring at the specific intensity for which an intermediate state is Stark shifted into multiphoton resonance. By graphing the electron yield as a function of laser intensity and fitting the data to a straight line on a log-log plot (slope of $\sim 5$ ), we are also able to estimate the multiphoton order for the resonant enhancement to be 5 .

Figure 2 shows a cartoon representation of two different level schemes which describe $5+2$ Freeman resonance enhanced multiphoton ionization (REMPI) into two separate electronic continua. For the scenario in the top portion of the figure, a single ground state $|0\rangle$ is coupled to a single excited state $|1\rangle$ by five photons. From $|1\rangle$, the system can be ionized to either continuum 1 or continuum 2 by a two photon transition. For the bottom scenario, $|0\rangle$ is now coupled with five photons to two intermediate states $|1\rangle$ and $|2\rangle .|1\rangle$ is only coupled to continuum 1 , and $|2\rangle$ is coupled only to continuum 2 , each by 2 photons.

The figure also illustrates how for a particular Stark shift of an intermediate state - and therefore for a particular intensity in the laser field - the ground state and intermediate state are brought into five-photon resonance. The rate of ionization into any continua that are coupled to this intermediate state is thus enhanced for this particular value of the intensity.

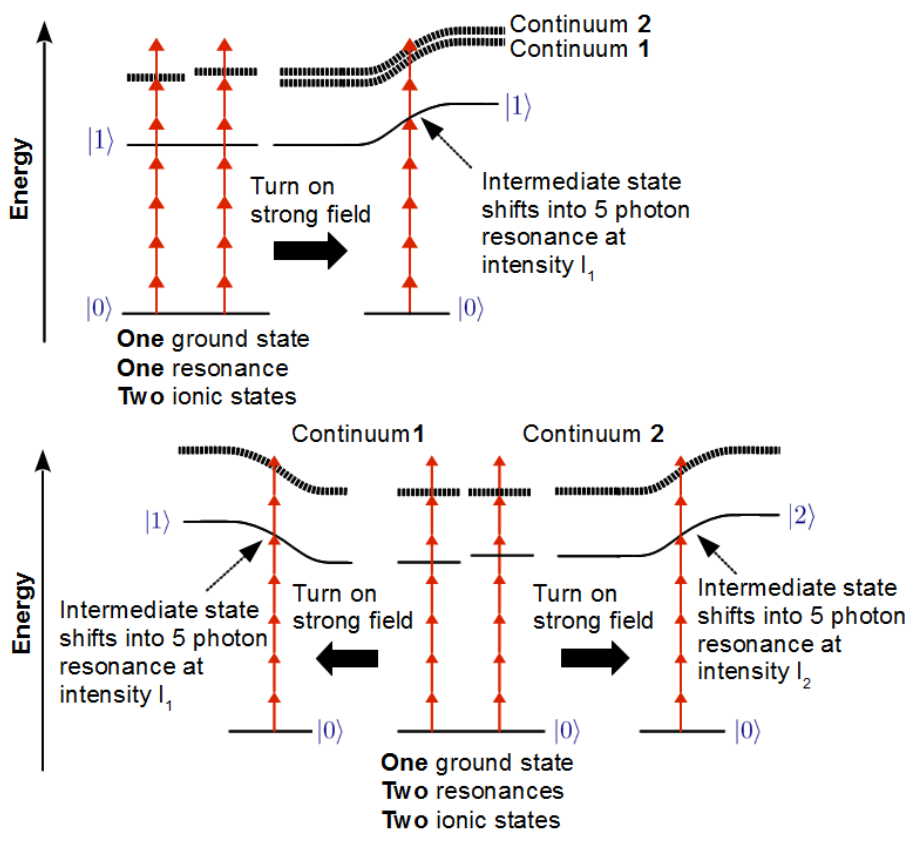

FIG. 2: Top: Cartoon diagram of $5+2$ ionization to two different ionic states. A single bound state Stark shifts into five-photon resonance to enhance ionization into both continua. Bottom: $5+2$ ionization to two different ionic states through two independent five-photon resonances. Resonance, in general, occurs at a different intensity for each intermediate state.

\section{THE MODEL}

In order to calculate the dependence of the photoelectron spectra on pulse shape for the two scenarios depicted in Figure 2, we integrated the time-dependent Schrödinger equation (TDSE) for a model system which we developed in order to describe the pulse shape dependence of strong field ionization involving Freeman resonances [15]. Our model is therefore very simple and ignores nuclear dynamics, molecular orientation and experimental issues such as focal volume averaging. It was designed to capture the role of dynamic Stark shifts and their interplay with pulse shaping in strong field ionization. In particular we consider a single ground state, one or two (intermediate) neutral excited states, which can be Stark shifted into multiphoton resonance with the ground state and two continua. The multiphoton couplings between these states are determined by considering the Schrödinger equation including all states of the system and then adiabatically eliminating all of nonresonant states [16]. The continuum states are included in our TDSE calculation by expanding the continuum photoelectron wavefunction, $\Psi_{\text {ion }}(E, t)$ in terms of Legendre polynomials of the kinetic energy as in $[15,17,18]$.

For all of our calculations, we started with a Gaussian laser pulse with a FWMH of $40 \mathrm{fs}$, a central frequency of $384 \mathrm{THz}$, and a peak intensity of $1.3 \times 10^{13} \mathrm{~W} / \mathrm{cm}^{2}$. 
We shaped the pulse by applying a $\pi$ phase flip in the frequency domain. Specifically, the spectral phase profile, $\phi(\omega)$, for a shaped pulse is given by:

$$
\phi(\omega)=\pi \Theta\left(\omega-\omega_{\text {flip }}\right)
$$

where $\Theta$ is the Heaviside function. We used a Bogacki-Shampine Runge-Kutta algorithm with an absolute precision $0.5 \times 10^{-9}$ to integrate the Schrödinger equation for an interval of 600 fs centered about the peak of an unshaped pulse. Given that the ionization potential of the molecule we consider here is $9.79 \mathrm{eV}$ [19], we consider 7 photon ionization. We performed calculations for intermediate states at 4,5 and 6 photon resonance, as well as calculations for 7 and 8 photon ionization and found that they all gave qualitatively similar results. As our intensity dependent yields showed a slope of 5 on a $\log -\log$ plot, we concentrate on the $5+2$ case, including a five-photon coupling between the ground state and intermediate state, and two-photon couplings from the intermediate state to the continua (as illustrated in figure 2). The initial field free detuning of the intermediate state was set to $125 \mathrm{THz}$ below five photon resonance, and the Stark shifts of the intermediate state and continua were all chosen such that the levels would shift by $132 \mathrm{THz}$ at the peak of the unshaped pulse. The initial detuning was chosen to be larger than the laser bandwidth and the Rabi frequencies involved, but significantly less than the laser frequency. The Stark shift of the intermediate neutral state at the peak of the laser pulse was chosen to be roughly equal to the experimentally determined ponderomotive shift The ponderomotive shifts were calibrated by measuring the photoelectron spectrum for $\mathrm{CS}_{2}$ (which displays pondermotively shifting peaks at the intensities used for the calibration) as a function of laser intensity. The multiphoton Rabi frequencies were chosen such that we obtained an ionization fraction consistent with experiment (between 0.01 and 0.1); the relative values of these couplings were chosen such that any population which moved into the intermediate state would subsequently move immediately into the continuum, and not undergo Rabi oscillations between the ground state and intermediate state. For the case of a two Freeman resonances (as shown schematically in the bottom panel of Figure 2), the initial detunings of the two intermediate states were set to $95 \mathrm{THz}$ and $125 \mathrm{THz}$. The Stark shifts of both intermediate states and both continua were again set so that the levels would shift upward by 132 $\mathrm{THz}$ at the peak intensity for an unshaped pulse. The multiphoton Rabi frequencies between the ground and intermediate states were set to be equal for the two intermediate states, while the intermediate to continuum Rabi frequencies were set to yield the same ratio between peaks as observed in experiments for an unshaped laser pulse.

\section{THE EXPERIMENTAL APPARATUS}

A detailed description of the laser system can be found in earlier publications [20]. It is based on a Kerr Lens modelocked Titanium Sapphire oscillator and a multipass amplifier, which generate near transform-limited laser pulses with a pulse duration of $30 \mathrm{fs}$ centered around $780 \mathrm{~nm}$, at a repetition rate of $1 \mathrm{kHz}$ and a maximum pulse energy of about $1 \mathrm{~mJ}$. The experiments described in this article utilize an ultrafast pulse shaper based on an acousto-optic modulator [21]. The laser pulses interact with the target molecule $\mathrm{CH}_{2} \mathrm{BrI}$ in a molecular beam chamber.

The vacuum chamber is equipped with a velocity map imaging lens and a spatially sensitive detector assembly (microchannel plates, phosphor screen and digital camera) similar to the one described in [22]. In addition, a $\mu$ metal enclosure mitigates the influence of stray magnetic fields. This apparatus allows us to measure the photoelectron momentum distribution after ionization. The raw camera images of the electron momentum distributions acquired by the camera were flat-field corrected by normalizing it with the response when the detector was illuminated by a de-focussed source of $\mathrm{H}^{+}$ions. This flatfield correction is important since depending on the age of the detector, the variation in detector gain across the microchannel plates can be as much as a factor of six. More details regarding the vacuum chamber are given in reference [23].

Energy calibration of the detector (the relationship between position on the detector and particle velocity/energy) was carried out by making use of the fact that the first and second above threshold ionization (ATI) peaks are spaced by the photon energy $(1.59 \mathrm{eV})$. We estimate a calibration accuracy of $7 \%$ or better. The laser intensity was determined by comparing the observed kinetic energy of photoelectrons for ionization to the first ionic state of $\mathrm{CS}_{2}$ with the expected photoelectron energy as given by the expression above for $\epsilon_{i}$ with $i=1$ [24]. If the differential AC Stark shift is neglected, the measured difference can be attributed to the ponderomotive energy and thus converted to an ionization intensity.

\section{RESULTS}

Calculations of the photoelectron spectra for the case of a single Freeman resonance and the case of two Freeman resonances were carried out for forty different $\pi$ phase flip frequencies spanning the range $\omega_{0}-15 \mathrm{THz}$ to $\omega_{0}+15 \mathrm{THz}$, where $\omega_{0}$ is the central frequency of the laser pulse. The results for the case of a single intermediate resonance are shown in the top panel of Figure 3, and the results for the case of two intermediate resonances are shown in the bottom panel of Figure 3. The top panel of figure 3 shows that if the two continua are coupled to the same intermediate state, no control is achieved as the $\pi$ phase flip frequency is varied. This is explicitly shown in 

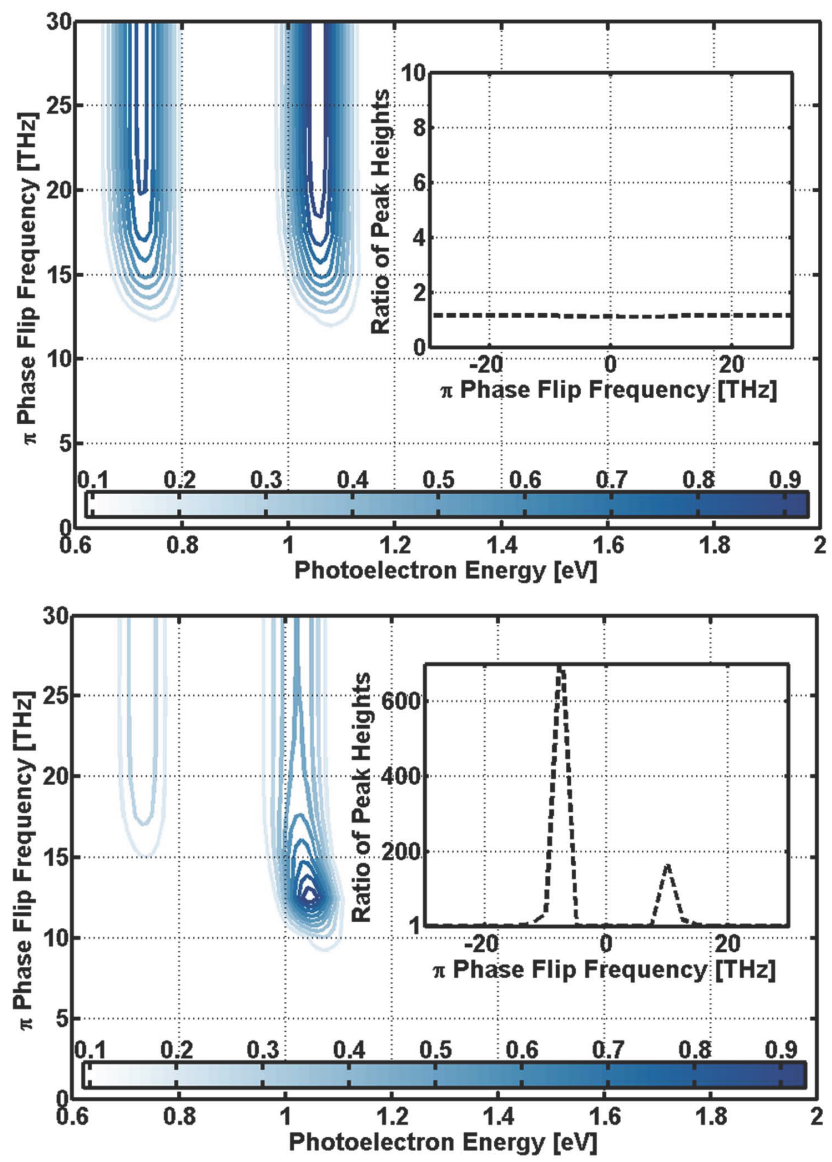

FIG. 3: Top: Simulated photoelectron spectrum (photoelectron yield vs photoelectron energy) as a function of $\pi$ phase flip frequency (measured from the central frequency) for a single intermediate resonance. The inset shows the ratio of the two peak heights as a function of flip frequency, normalized to the ratio of the peak heights for an unshaped pulse. The ratio remains constant, demonstrating a lack of control. Bottom: Simulated photoelectron spectrum as a function of photoelectron energy and $\pi$ phase flip frequency for the case of two separate intermediate resonances. The inset shows the ratio of the two peak heights, normalized to the ratio of the peak heights for an unshaped pulse. Here the ratio varies with $\pi$ phase flip frequency, demonstrating control over the relative ionic state populations. For both the top and bottom plots, the yield drops sharply for flip frequencies near the pulse's central frequency because the peak intensity for these shaped pulses is too low to shift the intermediate states into 5 photon resonance.

the inset plot, where the ratio of the two peak heights is plotted as a function of $\pi$ phase flip frequency. This result is consistent with the intuitive mechanism suggested by Figure 2: if the ionization rates for both continua are dependent on the same intermediate state shifting into five-photon resonance, then the variations in the photoelectron peaks for the two continua will be similar and little or no control is possible.

The bottom panel of figure 3 shows that if the two
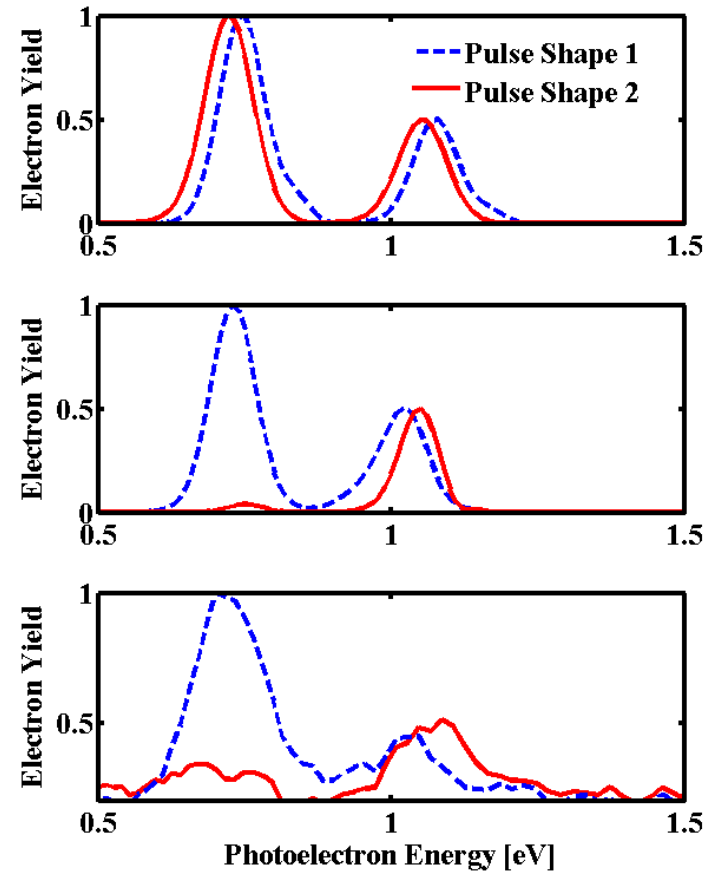

FIG. 4: Simulated and measured photoelectron spectra for two $\pi$ phase flip frequencies. Top: Simulated spectrum for two continua accessed through a single resonance. Middle: Simulated spectrum for two continua accessed through independent resonances. Bottom: Experimental spectra for $\mathrm{CH}_{2} \mathrm{BrI}$.

continua have separate intermediate resonances, then the relative heights of the two peaks in the photoelectron spectrum depend strongly on the $\pi$ phase flip frequency, demonstrating that significant control is achieved by means of varying the $\pi$ phase flip frequency. Calculations where we varied the laser intensity showed significantly less control than those where we varied the $\pi$ phase flip frequency.

The control dynamics here are very similar to the dynamics considered in earlier experiments and calculations, which focused on strong field multiphoton population transfer between neutral states in atomic sodium [25]. Shaping the pulse allows for matching the phase advance of the laser to the atom or molecule, whose levels are dynamically Stark shifted by the strong field of the laser pulse. Earlier work found that a pulse with a $\pi$ spectral phase jump was well suited to controlling the atom/molecule-laser phase, which motivated the use of this pulse shape parametrization in this work [26].

From Figure 3 we draw the conclusion that if control between two Freeman peaks is observed as the $\pi$ phase flip frequency is varied, then the two peaks must be associated with ionic states which are accessed through separate intermediate resonances. The top two panels of Figure 4 show the calculated photoelectron spectra for $\omega_{\text {flip }}=381 \mathrm{THz}$ and $369 \mathrm{THz}$ for the case of one Free- 
man resonance (top) and two Freeman resonances (middle). The bottom panel shows two Freeman peaks in the experimental photoelectron spectrum for $\mathrm{CH}_{2} \mathrm{BrI}$, plotted for two different $\pi$ phase flip positions. Control is clearly demonstrated in the experimental spectrum as the $\pi$ phase flip frequency is varied, suggesting that the two ionic states corresponding to these peaks are enhanced by separate intermediate states. Comparison of the bottom panel of Figure 4 with the top and middle panels clearly shows the correspondence between the experimental results and the model case of two Freeman resonances. We note that while the calculations shown here were carried out for $5+2$ ionization, similar calculations for $4+3$, $5+3,6+1$ and $6+2$ all yielded similar results, indicating that the results are not sensitive to the ionization or resonance multiphoton orders. Furthermore, we also carried out pulse shape dependent measurements with the molecules $\mathrm{CH}_{2} \mathrm{BrCl}$ and $\mathrm{CH}_{2} \mathrm{I}_{2}$, and we observed similar control over different Freeman resonance peaks in the photoelectron spectrum, indicating that the results are not specific or unique to $\mathrm{CH}_{2} \mathrm{BrI}$.

These results suggest that for the case of the two Freeman resonances considered here for $\mathrm{CH}_{2} \mathrm{BrI}$, each continuum is reached predominantly through a separate intermediate state - i.e. each neutral excited state is correlated with a single state of the cation. Similar observations of control in multiple molecules suggest that there are many cases of a neutral excited state being correlated with only one ionic state. While it is natural that a given neutral state should be correlated with a single ionic state in the case of weak field ionization[27], this has shown to be violated for strong field ionization from low lying neutral states [28, 29]. We argue that correlations between ionic states and higher lying, more weakly bound neutral excited states are stronger than those between ionic and low lying neutral excited states because for high lying states the electronic wavefunction is generally further from the core and thus the outgoing electron does not have as much opportunity to shakeup core electrons as it is removed from the molecule. Our observations of control, which implies some degree of correlation between neutral and ionic states, is consistent with correlations playing an important role in Freeman resonance enhanced multiphoton ionization when the resonance enhancement comes from high lying neutral states, $\mathrm{S}_{n}(n \gg 1)$ well above the ground state.

\section{CONCLUSION}

In conclusion, a comparison between experimental measurements of the photoelectron spectrum resulting from pulse shape dependent strong field molecular ionization with simple calculations that model Freeman resonance mediated strong field ionization allows us to determine whether intermediate resonances are common to separate electronic continua or not. The fact that we can control the ratio of electrons associated with different final ionic states via pulse shaping indicates that separate intermediate resonances enhance the ionization to separate ionic states. As this behavior is observed in several molecules, it suggests that high lying neutral states correlate well with only a single ionic continuum in the case of strong field ionization.

\section{ACKNOWLEDGMENTS}

The authors gratefully acknowledge insightful discussions with Michael Spanner and support from the National Science Foundation under award number 1205397.
[1] A. E. Boguslavskiy, J. Mikosch, A. Gijsbertsen, M. Spanner, S. Patchkovskii, N. Gador, M. J. J. Vrakking, and A. Stolow, Science 335, 1336 (2012).

[2] D. Geißler, T. Rozgonyi, J. González-Vázquez, L. González, S. Nichols, and T. Weinacht, Physical Review A 82 (2010).

[3] H. Akagi et al., Science 325, 1364 (2009).

[4] G. N. Gibson, R. R. Freeman, and T. J. McIlrath, Physical Review Letters 67, 1230 (1991).

[5] S. Haessler, J. Caillat, W. Boutu, C. Giovanetti-Teixeira, T. Ruchon, T. Auguste, Z. Diveki, P. Breger, A. Maquet, B. Carré, et al., Nature Physics 6, 200 (2010).

[6] F. Krausz and M. Ivanov, Rev. Mod. Phys. 81, 163 (2009).

[7] M. Kling and M. Vrakking, Annu. Rev. Phys. Chem. 59, 463 (2008).

[8] I. V. Hertel and W. Radloff, Reports on Progress in Physics 69, 1897 (2006).

[9] M. V. Ammosov, N. B. Delone, and V. P. Krainov, Sov. Phys. JETP 64, 1191 (1986).
[10] L. V. Keldysh, Sov. Phys. JETP 20, 1307 (1965).

[11] R. R. Freeman and P. H. Bucksbaum, Journal of Physics B: Atomic, Molecular and Optical Physics 24, 325 (1991).

[12] M. Spanner, S. Patchkovskii, C. Zhou, S. Matsika, M. Kotur, and T. C. Weinacht, Phys. Rev. A 86, 053406 (2012).

[13] J. González-Vázquez, L. González, S. R. Nichols, T. C. Weinacht, and T. Rozgonyi, Physical Chemistry Chemical Physics 12, 14203 (2010).

[14] P. Sándor, X. Zhao, and T. Rozgonyi, T. Weinacht, Journal of Physics B - to be published (2013).

[15] W. D. Lunden, P. Sándor, T. Rozgonyi, and T. C. Weinacht, submitted (2013).

[16] C. Trallero-Herrero, D. Cardoza, T. C. Weinacht, and J. L. Cohen, Phys. Rev. A 71, 013423 (2005).

[17] M. Seel and W. Domcke, Journal of Chemical Physics 95, 7806 (1991).

[18] T. Rozgonyi, A. Glass, and T. Feurer, Journal of Applied Physics 88, 2936 (2000).

[19] A. F. Lago, J. P. Kercher, A. Bödi, B. Sztáray, B. Miller, 
D. Wurzelmann, and T. Baer, The Journal of Physical Chemistry A 109, 1802 (2005).

[20] F. Langhojer, D. Cardoza, M. Baertschy, and T. Weinacht, The Journal of chemical physics 122, 014102 (2005).

[21] M. A. Dugan, J. X. Tull, and W. S. Warren, Journal of the Optical Society of America B 14, 2348 (1997).

[22] A. T. J. B. Eppink and D. H. Parker, Review of Scientific Instruments 68, 3477 (1997).

[23] D. Geißler, T. Rozgonyi, J. González-Vázquez, L. González, P. Marquetand, and T. C. Weinacht, Physical Review A 84, 053422 (2011).

[24] A. W. Potts and G. H. Fattahallah, Journal of Physics B: Atomic and Molecular Physics 13, 2545 (1980).
[25] C. Trallero-Herrero, J. L. Cohen, and T. Weinacht, Phys. Rev. Lett. 96, 063603 (2006).

[26] C. Trallero-Herrero and T. C. Weinacht, Phys. Rev. A 75, 063401 (2007).

[27] B. T. Pickup, Chemical Physics 19, 193 (1977), ISSN 0301-0104.

[28] M. Kotur, T. C. Weinacht, C. Zhou, S. Matsika, S. Patchkovskii, and M. Spanner, Physical Review Letters 109, 203007 (2012).

[29] M. Spanner, S. Patchkovskii, C. Zhou, S. Matsika, M. Kotur, and T. C. Weinacht, Physical Review A 86, 053406 (2012). 